\title{
Pain-relieving effect of a compound isolated from white peony root oral liquid on acute radiation-induced esophagitis
}

\author{
ZHIYU WANG ${ }^{1}$, LI SHEN $^{2}$, XING LI $^{1}$, XIN SHU ${ }^{3}$, BAOEN SHAN $^{1}$, LI ZHANG $^{1}$, \\ YANJUN GONG ${ }^{4}$ and ZHIMING DONG $^{1}$ \\ ${ }^{1}$ Department of Biotherapy, The Fourth Hospital of Hebei Medical University, Shijiazhuang, Hebei 050011; \\ ${ }^{2}$ Department of Traditional Chinese Medicine, The First Hospital of Hebei Medical University, Shijiazhuang, Hebei 050031; \\ ${ }^{3}$ Department of Medical Oncology, The General Hospital of Chinese Armed Police Force, Haidian, Beijing 100039; \\ ${ }^{4}$ Centre of HMO Administrator of Shijiazhuang, Shijiazhuang, Hebei 050031, P.R. China
}

Received December 20, 2012; Accepted April 8, 2013

DOI: $10.3892 / \mathrm{mmr} .2013 .1427$

\begin{abstract}
Acute radiation-induced esophagitis (ARIE) is a common complication of radiotherapy. The aim of this study was to clarify the molecular mechanism of pain relief by the compound of white peony root oral liquid (cWPROL) in ARIE. An animal model of ARIE was established and either cWPROL or a mixture of lidocaine, dexamethasone and gentamycin (mLDG) was administered. We indirectly observed rat symptoms of pain by recording the weight of food and the volume of water consumed by the rats, along with changes in body weight. Additionally, the expression levels of substance P (SP) in the esophageal tissues were detected by immunohistochemistry. It was demonstrated that cWPROL was able to release the pain of ARIE by decreasing the expression of SP; this may be one of the molecular mechanisms via which cWPROL induces pain relief.
\end{abstract}

\section{Introduction}

A large proportion of patients with thoracic carcinomas receive thoracic radiotherapy (TRT) as a part of their treatment. A number of these patients exhibit radiation-induced toxicity, such as acute radiation-induced esophagitis (ARIE), which is the primary dose-limiting complication of radiotherapy. The incidence rate of ARIE in a clinical setting is relatively high and the common symptoms are non-specific, mainly comprising dysphagia, odynophagia and a substernal burning sensation (1). As demonstrated in Table I, the degrees of ARIE defined by the National Cancer Institute Common Toxicity Criteria (NCI-CTC) (2) are essentially based on the symptoms patients have experienced. Clinically, patients often complain

Correspondence to: Dr Zhiyu Wang, Department of Biotherapy, The Fourth Hospital of Hebei Medical University, 12 Jian Kang Road, Shijiazhuang, Hebei 050011, P.R. China

E-mail: drwangzhiyu@hotmail.com

Key words: acute radiation-induced esophagitis, compound of white peony root oral liquid, substance $\mathrm{P}$, pain of dysphagia, odynophagia and a substernal burning sensation 2-3 weeks after initiation of radiotherapy (2). The course of radiation treatment is delayed if complications are serious.

Cell death and defluxion of the esophageal mucous epithelium in the radiation fields have been observed, along with bleeding or ulceration when the dose of radiation was sufficiently high (3). Inflammatory cell infiltration in the esophageal tissue is another prominent histopathological change that occurs in ARIE $(3,4)$. These types of damage may evoke symptoms of pain, and substance P (SP) secretion may be increased, playing an important role in the inflammatory procedure. SP is an 11 amino acid polypeptide released by sensory nerves. The biological function of SP is induced by its binding to the receptor, neurokinin-1 (5), which is expressed on a limited number of cell types, including epithelial cells, fibroblasts and inflammatory cells (6). As a neurotransmitter and a neuromodulator, SP is associated with inflammatory processes and pain. It participates in the process of nociception by transmitting information regarding damage from the peripheral receptors to the central nervous system, thus resulting in the generation of symptoms of pain (7).

In the present study, we established the animal model of ARIE and demonstrated the therapeutic effect of the compound of white peony root oral liquid (cWPROL) on this model (8). We elucidated a molecular mechanism via which the symptoms of pain are relieved; cWPROL is able to relieve symptoms of pain in the ARIE animal model by decreasing the expression level of SP in esophageal tissues.

\section{Materials and methods}

Subjects. Adult male Wistar rats with an average weight of 180-220 g were used. Animals were housed with a 12-hour light/dark cycle, and were given access to food and water ad libitum. All experimental animal techniques and handling procedures were approved by the Institutional Animal Care and Use Committee of Hebei Medical University. The certification number of the animals was DK0512053.

Grouping and irradiation. Twenty-four subjects were randomly divided into four groups $(n=6)$, including the 
cWPROL treatment (CT) group; the mixture of lidocaine, dexamethasone and gentamycin (mLDG) treatment (MT) group; the radiation (R) group; and the non-intervention (NI) group. In the CT group, rats were administered $0.475 \mathrm{~g} / \mathrm{ml}$ cWPROL by intra-esophageal perfusion following irradiation; while in the MT group, rats were treated with $\mathrm{mLDG}$, which was also administered following irradiation. By contrast, in the $\mathrm{R}$ group, rats were not administered any treatment following irradiation; while in the NI group, rats did not receive irradiation or treatment. Animals receiving treatment were administered $2 \mathrm{ml}$ of the agent, 3 times a day, with treatment initiated on the seventh day following irradiation and continuing for 7 days. Rats were deprived of food and water for $30 \mathrm{~min}$ following administration.

Conscious rats received a single dose of gamma irradiation (total dose, $43 \mathrm{~Gy}$ ) to the chest. The irradiation procedure was performed with a ${ }^{60} \mathrm{Co}$ therapy apparatus (Xinhua Factory, Shandong, China) at a dose rate of $0.111 \mathrm{~Gy} / \mathrm{min}$. The irradiation field was $3 \times 30 \mathrm{~cm}$, with a center dose point on the back of the rats located $1 \mathrm{~cm}$ beneath the body surface, and an irradiation range of $3 \mathrm{~cm}$ on the upper esophagus; the remaining parts of the body were covered. Rats in the NI group were not irradiated, but were otherwise treated as irradiated rats.

Detection of the general condition of the rats. Before and after the experiment, rat body weights in each group were recorded, in addition to the daily weight of food and volume of water. These data were regarded as indirect evidence reflecting symptoms of pain.

Immunohistochemical staining. Rats were anesthetized with $2 \%$ pentobarbital sodium administered by intraperitoneal injection $(45 \mathrm{mg} / \mathrm{kg}$ ). Esophageal samples were fixed with $4 \%$ paraformaldehyde for $24 \mathrm{~h}$, embedded in paraffin and sectioned at $4 \mu \mathrm{m}$. Tissue sections were deparaffinized with xylene, rehydrated through an ethanol series and with TBST and immersed in $3 \%$ formaldehyde hydrogen peroxide liquid to block endogenous peroxidase. Antigen retrieval was performed by microwave treatment in the presence of antigen retrieval solution. The sections were incubated with the primary antibody at $4^{\circ} \mathrm{C}$ overnight, and then treated with biotin-labeled secondary antibody (Zhongshan Jinqiao Bio-technology Limited Company, Beijing, China) at $37^{\circ} \mathrm{C}$ for $20 \mathrm{~min}$. This was followed by the addition of a peroxidase-conjugated streptavidin-labeled tertiary antibody (Zhongshan Jinqiao Bio-technology Limited Company) at $37^{\circ} \mathrm{C}$ for $20 \mathrm{~min}$. We used antibodies against SP (1:50) as the primary antibodies (Boshide Program Limited Company, Wuhan, China). The sections were counterstained with hematoxylin, dehydrated, transparentized and then sealed with neutral gum. The blank control and replacing control were treated with PBS and normal rabbit serum, respectively. The appearance of brown particles in the stained parts was regarded as positive. Five successive visual fields centered on the lesion area of each section were observed under a microscope $(\mathrm{x} 400)$ and the average of their integral optical density (IOD) was regarded as the representative value.

Statistical analysis. Experimental results were analyzed for their statistical significance using SPSS 13.0 software (SPSS,
Table I. Grade of acute radiation-induced esophagitis.

\begin{tabular}{ll}
\hline Grade & \multicolumn{1}{c}{ Description } \\
\hline 0 & None \\
1 & $\begin{array}{l}\text { Mild dysphagia, but is able to eat regular diet } \\
2\end{array}$ \\
$\begin{array}{l}\text { Dysphagia, requiring predominantly pureed, soft or } \\
\text { liquid diet }\end{array}$ \\
$\begin{array}{l}\text { Dysphagia, requiring feeding tube, i.v. hydration } \\
\text { or hyperalimentation } \\
\text { Complete obstruction (cannot swallow saliva); } \\
\text { ulceration with bleeding not induced by minor } \\
\text { trauma, abrasion or perforation }\end{array}$
\end{tabular}

Inc., Chicago, IL, USA). Groups were compared by a one-way ANOVA. The Student-Newman-Keuls test was utilized when the variance was equal, while the Kruskal-Wallis $\mathrm{H}$ test was used if the variance was unequal. Results are presented as the mean \pm standard deviation. $\mathrm{P}<0.05$ was considered to indicate a statistically significant difference.

\section{Results}

The effect of treatment on rat body weight. As demonstrated in Fig. 1, there was no significant difference in body weight between groups prior to the experiment $(\mathrm{P}>0.05)$. Following the experiment, the body weight of rats in the NI group significantly increased $(\mathrm{P}<0.05)$; while that of the remaining experimental groups significantly decreased $(\mathrm{P}<0.05)$, particularly in the MT group $(\mathrm{P}<0.01)$. Additionally, compared with the $\mathrm{R}$ group, there was no significant difference in the body weight of rats in the CT group, while the body weight significantly decreased in the MT group $(\mathrm{P}<0.05)$. Furthermore, the body weight of rats in the MT group was significantly lower than that of the CT group $(\mathrm{P}<0.05)$.

Changes in the weight of food and volume of water following treatment. We detected the daily changes in the weight of food and volume of water, and these were assumed to indirectly reflect the symptoms of pain. As demonstrated in Fig. 2, the weight of food and volume of water remained stable in the NI group, while those of the remaining experimental groups gradually decreased and reached the lowest point on the seventh day following irradiation, before slowly increasing. We found that following administration of cWPROL or $\mathrm{mLDG}$, the volume of water was increased to a certain extent compared with that of the R group. Notably, compared with the R group, the weight of food rats consumed in the MT group did not increase, thus demonstrating that the symptoms of pain in this group were not effectively reduced.

The level of SP expression in each group. We observed the variation in the expression levels of SP in the esophageal tissues by immunohistochemistry, before and after irradiation and treatment administration (Fig. 3). It was revealed that the expression level of SP was increased following exposure to radiation; in the $\mathrm{R}$ group, the expression of SP was significantly 
- before the experiment $\square$ after the experiment

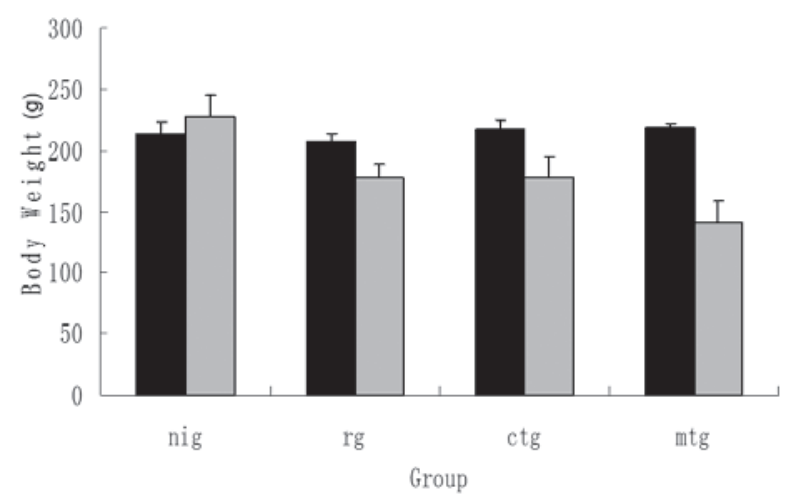

Figure 1. Change in body weight in each group. Nig, non-intervention group; $\mathrm{rg}$, radiation group; ctg, the compound of white peony root oral liquid (cWPROL) treatment group; $\mathrm{mtg}$, the mixture of lidocaine, dexamethasone and gentamycin (mLDG) treatment group.

increased compared with the NI group $(\mathrm{P}<0.01)$. A low level of SP expression was observed in normal rat esophageal tissues, and was mainly distributed in the mucosal epithelium and nerve fiber peripheries (Fig. 3A). Following irradiation in the $\mathrm{R}$ group, marked SP expression was detected in the epithelium of the area surrounding ulcers, the fibrocytes and fibroblasts of the lamina propria and the submucosa in the inflamed area (Fig. 3B). Following the administration of cWPROL, the expression of SP was lower than that of the R group $(\mathrm{P}<0.01)$, but higher than that of the NI group $(\mathrm{P}<0.05)$; while the location of SP had no clear variance (Fig. 3C). The result of the MT group was as predicted; the expression level of SP in the MT group was significantly different compared with that of the NI $(\mathrm{P}<0.01)$ and $\mathrm{R}(\mathrm{P}<0.05)$ groups (Fig. 3D). With regard to the comparison between the $\mathrm{CT}$ and MT groups, although the difference in SP expression levels was not significantly different, the effect of reducing the expression level of SP by administration of cWPROL was greater than that of $\mathrm{mLDG}$ $(\mathrm{P}>0.05)$.

\section{Discussion}

$\mathrm{SP}$ is a member of the tachykinin family that is secreted by neurons and other types of cells. SP is also detected in endothelial cells, inflammatory cells, fibroblasts and other types of cells (9). It has a wide range of functions, which include inducing cancer cell proliferation, such as in malignant melanomas (10). In the present study, we focused on the mediating function of SP, which involves delivering nociceptive information to the nervous system. It is commonly known that SP acts through the tachykinin NK1 receptor, which is a member of the G-protein-coupled receptor superfamily. Additionally, Bie and Zhao (11), along with Holzer (12), have demonstrated that the tachykinin NK1 receptor may contribute to the development and maintenance of inflammatory pain as a nociceptor. It has been demonstrated that SP is upregulated in response to irradiation and is involved in the process of inflammation (13). Therefore, we hypothesized that SP may also influence the symptoms of pain caused by radiation-induced inflammation. In addition, secreted SP may promote the inflammatory procedures caused by radiation, through facilitating inflammatory cell infiltration and activation (14).

The aim of radiation treatment is to administer an effective dose of radiation to the tumor, with an acceptable dose delivered to the neighboring normal tissues; however, the radiotherapy of thoracic neoplasms is likely to expose the esophagus to a high dose of ionizing radiation. In clinical settings, ARIE is a common complication in cancer patients receiving radiotherapy. Among these patients, a large proportion suffer from serious symptoms of pain and their quality of life is reduced. Furthermore, certain patients are forced to suspend their irradiation treatment when they are not able to tolerate any further treatment, resulting in a decreased tumor control rate. As for the treatment of ARIE, there are currently no clinically effective drugs or strategies. Minimizing the extent of the esophagus that is irradiated is an effective means to prevent ARIE; however, this would reduce the control of thoracic malignancies. Adrenocorticotropic hormones and certain antibiotics are the main drugs used in the treatment of ARIE; however, few efficacies have been demonstrated in a wide range of patients $(4,15)$. Furthermore, the adverse effects of these drugs, including an increased risk of osteoporosis and antibiotic resistance, negatively limit their usage. Studies have also investigated the effect of sucralfate on preventing ARIE; however, $58 \%$ of patients resigned from the study, due to the adverse effects of nausea and vomiting (16). Another drug, amifostine, regarded to be the most effective radioprotective compound screened by the U.S. Army, did not effectively relieve the symptoms of ARIE in a large clinical trial (RTOG 9801) (17). Therefore, there is a requirement to investigate a novel method with effective functions and without obvious side-effects.

Drugs of a herbal origin with few side effects are of high interest amongst alternative treatments. Furthermore, traditional Chinese herb medicine (tChm) may provide a novel therapy that is not only able to relieve clinical symptoms, but also improve the general condition, including eating, sleeping and immune function $(18,19)$. We have previously investigated the effect of cWPROL in treating the animal model of ARIE, and demonstrated that this prescription formula was able to repair the damaged esophageal tissues (20). The present study was designed to demonstrate that cWPROL is able to relieve the symptoms of pain caused by irradiation, and to clarify the underlying mechanism. We observed the general conditions, including body weight, and the weight of food and volume of water, to indirectly reflect symptoms of pain. Meanwhile, the expression level of SP in tissues within the radiation field was detected using immunohistochemical staining. As demonstrated by the results, cWPROL was able to decrease the level of SP expression, which was hypothesized to be correlated with a decrease in the symptoms of pain. Correspondingly, in the CT group, the weight of food and volume of water increased to a certain extent, after the lowest point of weight of food on the seventh day and the lowest point of volume of water on the eighth day. However, in the MT group, the weight of food and volume of water did not increase, although decreased SP expression was observed. It may be concluded that the pain relieving effect of $\mathrm{mLDG}$ is not as strong as that of cWPROL. 

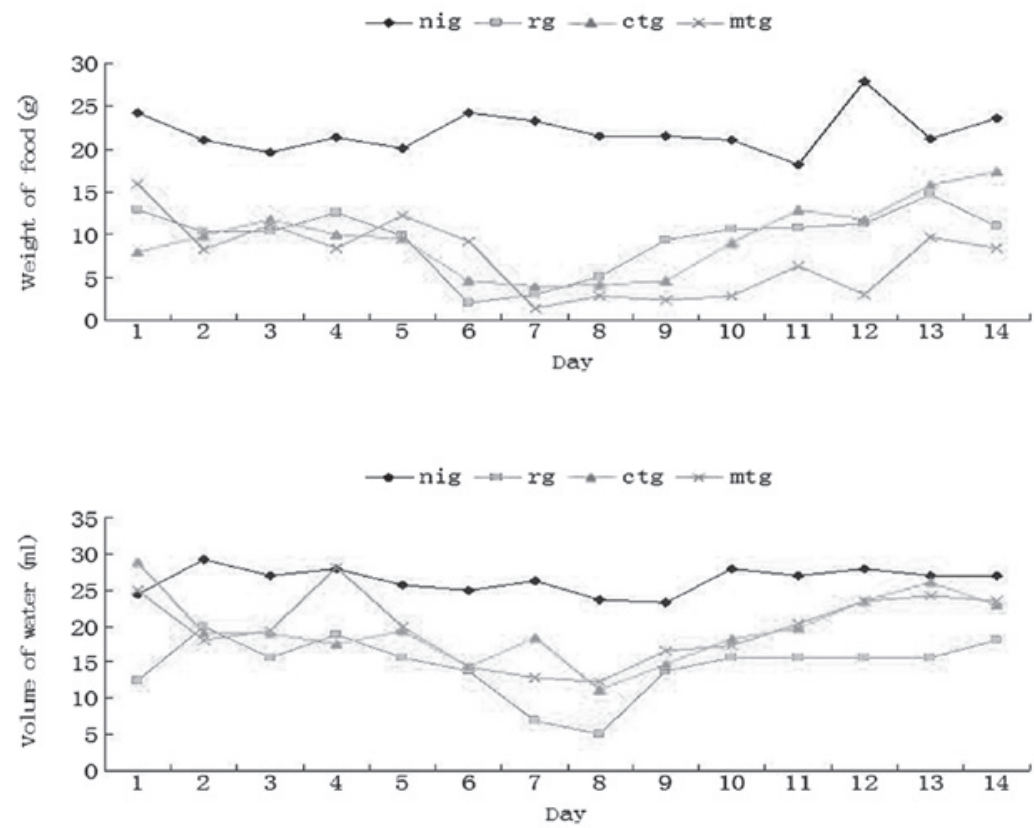

Figure 2. Changes in weight of food and volume of water following treatment administration. Nig, non-intervention group; rg, radiation group; ctg, the compound of white peony root oral liquid (cWPROL) treatment group; mtg, the mixture of lidocaine, dexamethasone and gentamycin (mLDG) treatment group.

A

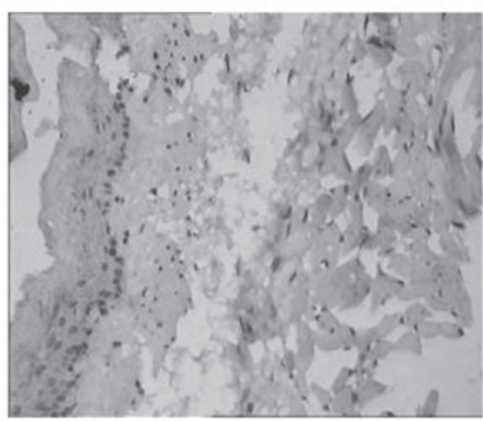

C

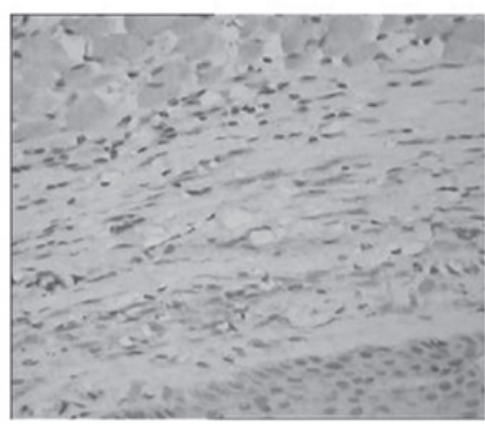

B

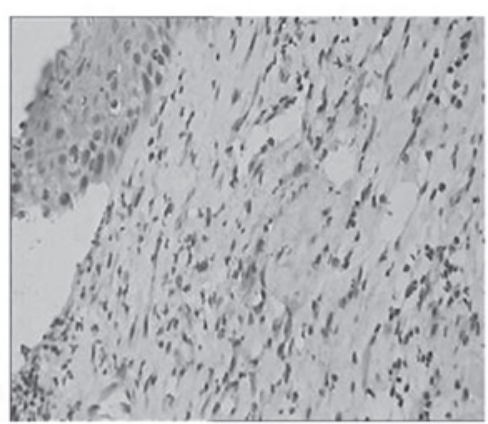

D

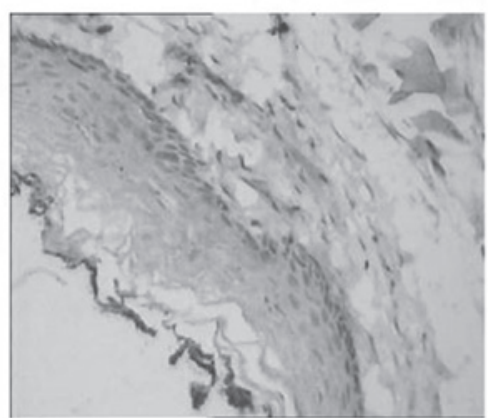

\begin{tabular}{|c|c|c|c|c|}
\hline Group & $\operatorname{nig}(\mathrm{A})$ & $\mathrm{rg}(\mathrm{B})$ & $\operatorname{ctg}(\mathrm{C})$ & $\mathrm{mtg}(\mathrm{D})$ \\
\hline IOD & $5.12 \pm 1.56$ & $17.37 \pm 5.14$ & $9.13 \pm 4.46$ & $11.73 \pm 4.54$ \\
\hline
\end{tabular}

Figure 3. Expression level of substance $\mathrm{P}$ (SP) in each group ( $\mathrm{x} 400$, immunohistochemistry). (A) Normal rat esophageal tissue in nig; (B) rat esophageal tissue in rg; (C) rat esophageal tissue in ctg; (D) rat esophageal tissue in mtg. Nig, non-intervention group; rg, radiation group; ctg, the compound of white peony root oral liquid (cWPROL) treatment group; mtg, the mixture of lidocaine, dexamethasone and gentamycin (mLDG) treatment group.

According to the outcomes of the present study, a partial mechanism for the pain-relieving effect of cWPROL has been elucidated. SP plays a key role in delivering pain information to the nervous system when tissues respond to injury (9). Our study suggests that the expression level of SP may be reduced by administration of cWPROL, and that the general condition, including body weight and the intake of food or drinking water, may be improved to a certain extent. However, other 
detailed molecular mechanisms should also be investigated in more detail in the future. For example, the critical receptor of SP, the tachykinin NK1 receptor, may exert a simultaneous influence.

\section{Acknowledgements}

This study was supported by the Foundation Science Research Program of Hebei Province Science and Technology Office (no. 04236101D-252004-2005).

\section{References}

1. Byhardt RW, Scott C, Sause WT, et al: Response, toxicity, failure patterns, and survival in five Radiation Therapy Oncology Group (RTOG) trails of sequential and/or concurrent chemotherapy and radiotherapy for locally advanced non-small-cell carcinoma of the lung. Int J Radiat Oncol Biol Phys 42: 469-478, 1998.

2. Bradley J and Movsas B: Radiation esophagitis: Predictive factors and preventive strategies. Semin Radiat Oncol 14: 280-286, 2004

3. Abdel-Latif MM, Duggan S, Reynolds JV and Kelleher D: Inflammation and esophageal carcinogenesis. Curr Opin Pharmacol 9: 396-404, 2009.

4. Liu Y, Yu H, Zhang C, Cheng Y, Hu L, Meng X and Zhao Y: Protective effects of berberine on radiation-induced lung injury via intercellular adhesion molecular-1 and transforming growth factor-beta-1 in patients with lung cancer. Eur J Cancer 44: 2425-2432, 2008

5. Yamamoto K, Kureyama N, Asano K, Ikeda T and Yamatodani A: Involvement of substance $\mathrm{P}$ and the neurokinin-1 receptor in radiation-induced hair loss in mice. J Pharmacol Sci 112: $118-120,2010$

6. Wang $\mathrm{J}$ and Hauer-Jensen M: Neuroimmune interactions: potential target for mitigating or treating intestinal radiation injury. Br J Radiol 80: S41-S48, 2007.

7. Yasui Y, Saper CB and Cechetto DF: Calcitonin gene-related peptide immunoreactivity in the visceral sensory cortex, thalamus, and related pathways in the rat. J Comp Neurol 290: 487-501, 1989.
8. Li S, Baoen S, Li Z, et al: Establishment of animal model of radiation esophagitis. Chin J Cancer Prev Treat 14: 13-16, 2007.

9. Nessler S, Stadelmann C, Bittner A, et al: Suppression of autoimmune encephalomyelitis by a neurokinin-1 receptor antagonist - a putative role for substance $\mathrm{P}$ in CNS inflammation. J Neuroimmunol 179: 1-8, 2006.

10. Korcum AF, Sanlioglu S, Aksu G, Tuncel N and Erin N: Radiotherapy-induced decreases in substance P levels may potentiate melanoma growth. Mol Med Rep 2: 319-326, 2009.

11. Bie BH and Zhao ZQ: Peripheral inflammation alters desensitization of substance P-evoked current in rat dorsal root ganglion neurons. Eur J Pharmacol 670: 495-499, 2011.

12. Holzer P: Implications of tachykinins and calcitonin gene-related peptide in inflammatory bowel disease. Digestion 59: 269-283, 1998.

13. Christensen HD and Haley TJ: Distribution of substance P in the central nervous system and small intestine of the rat after x-irradiation. Radiat Res 33: 588-595, 1968

14. Foster AP and Cunningham FM: Substance $P$ induces activation, adherence and migration of equine eosinophils. J Vet Pharmacol Ther 26: 131-138, 2003.

15. Kosaka Y, Mitsumori M, Araki N, et al: Avascular necrosis of bilateral femoral head as a result of long-term steroid administration for radiation pneumonitis after tangential irradiation of the breast. Int J Clin Oncol 11: 482-486, 2006.

16. McGinnis WL,Loprinzi CL, Buskirk SJ, et al: Placebo-controlled trail of sucralfate for inhibiting radiation-induced esophagitis. J Clin Oncol 15: 1239-1243, 1997.

17. Movsas B, Scott C, Langer C, et al: Phase III study of amifostine in patients with locally advanced non-small cell lung cancer (NSCLC) receiving chemotherapy and hyperfractionated radiation (chemo/HFxRT): Radiation Therapy Oncology Group (RTOG) 98-01. Proc Am Soc Clin Oncol 22 (abstr 2559): 636, 2003.

18. Hou W and Zhou Y: Function of traditional Chinese medicine in cancer radiotherapy and its prospect. World Sci Tech 11: 742-746, 2009.

19. Zhang P and Hu PL: TCM VVM Therapy's influence on tumor patients' survival. Chin J Oncol 25: 302, 2003.

20. Shen L, Shan BE, Zhang L, et al: Study on preventive and therapeutic function of compound white pony root oral liquid in treating radiation-induced esophagitis. Radiat Protect 27: 119-227, 2007. 